\title{
Red Galaxies from Hot Halos in Cosmological Hydro Simulations
}

\author{
Jared Gabor ${ }^{1}$ \\ ${ }^{1}$ CEA Saclay \\ Bat. 709, Gif-sur-Yvette, 91191 France \\ email: jared.gabor@cea.fr
}

\begin{abstract}
I highlight three results from cosmological hydrodynamic simulations that yield a realistic red sequence of galaxies: 1) Major galaxy mergers are not responsible for shutting off star-formation and forming the red sequence. Starvation in hot halos is. 2) Massive galaxies grow substantially $(\sim \times 2$ in mass) after being quenched, primarily via minor (1:5) mergers. 3 ) Hot halo quenching naturally explains why galaxies are red when they either (a) are massive or (b) live in dense environments.
\end{abstract}

Keywords. galaxies: evolution, galaxies: interactions

\section{Major mergers are not responsible for quenching}

The physical mechanism(s) responsible for shutting down star-formation and leading to the red sequence are still debated (Bower et al. 2006, Croton et al. 2006, Hopkins et al. 2008, Somerville et al. 2008). Two leading pictures have emerged: a) major mergers trigger starbursts and possibly AGN, whose combined gas consumption and feedback can rid the galaxy of gas to fuel star-formation; and b) hot gas coronae form in massive halos, shock-heating any infalling gas, and some heating process (such as a radio AGN) prevents that gas from cooling. Often, these are respectively called "quasar" mode and "radio" mode AGN feedback, alluding to the possible importance of AGN.

I have independently tested simplistic versions of these two mechanisms in cosmological hydrodynamic simulations. For merger quenching, I identify mergers on-the-fly during the simulation and eject all the gas from remnants in a $1000 \mathrm{~km} \mathrm{~s}^{-1}$ wind. For hot halo quenching, I identify galaxies whose halos are dominated by hot gas $\left(T>10^{5.4} \mathrm{~K}\right.$; Kereš et al. 2005), and continuously add thermal energy to the circum-galactic gas around them. The results of these simulations are shown in Figure 1 (cf. Gabor et al. 2011) as color-magnitude diagrams and luminosity functions. Merger quenching fails to yield a significant red sequence at $z=0$, whereas hot halo quenching forms red galaxies in numbers consistent with observations.

In the context of cosmological models, major mergers are neither necessary nor sufficient to explain the red sequence. They are not sufficient because galaxies are constantly accreting new gas from the cosmic web, even after mergers. Since the accreted gas provides fuel for star-formation, this accretion must be stopped to ensure that galaxies become red and stay red. Mergers are not necessary because an alternative quenching mechanism - hot halo quenching - appears to produce enough red galaxies. Note that there are enough major mergers to explain the numbers of red galaxies, if only the galaxies stopped accreting after the merger (Gabor et al. 2010). 

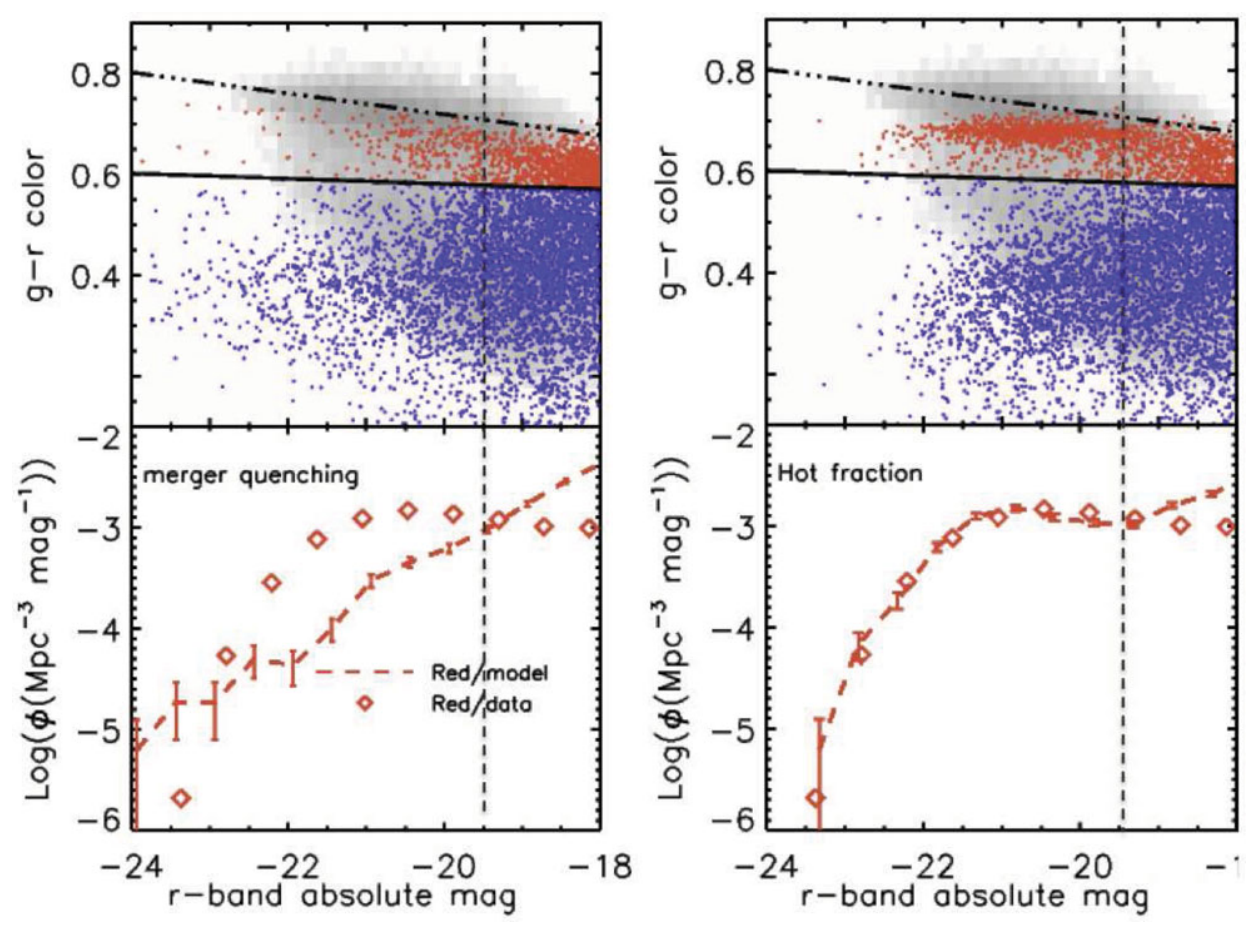

Figure 1. Merger quenching (left) does not produce enough red galaxies, but hot gas quenching (right) does. In color-magnitude diagrams (top panels) colored points represent simulated galaxies, and the grayscale represents SDSS galaxies. In the luminosity functions (bottom panels), dashed lines with error bars show the number densities of simulated red galaxies, and diamonds those from SDSS. Only hot halo quenching matches the $z \sim 0$ number densities of red galaxies. Based on Gabor et al. (2011).

\section{Massive galaxies grow substantially after quenching}

In the hot halo quenching simulation, massive galaxies typically turn red at $z>0.5$, and grow significantly in mass after being quenched. The mass growth is shown in the left panel of Figure 2. Since star-formation is negligible in these galaxies, there are only two ways to change in stellar mass: mass loss from stellar evolution, and galaxy mergers. By number, most galaxies do not change much in mass - these are mostly recently-quenched satellites which are unlikely to merge with other satellites. Massive galaxies, on the other hand, grow by factors up to 3 , with a large scatter driven by variations in merger history.

These massive galaxies are typically central galaxies accreting their small satellites in minor mergers. The right-hand panel of Figure 2 shows the mean merger mass ratio (for those galaxies with at least one merger) as a function $z=0$ stellar mass. Here I have weighted each merger event by the mass of the smaller galaxy. Thus the plot shows the mass ratio which has been most important for adding mass to galaxies in each bin of stellar mass. In all cases, the characteristic merger is below the typical 1:3 major merger threshold, with a typical value of 1:5. Minor mergers dominate the mass growth of quenched galaxies. This result implies strong growth in the sizes of quenched galaxies at high redshift (Gabor et al. 2012, Oser et al. 2012). 

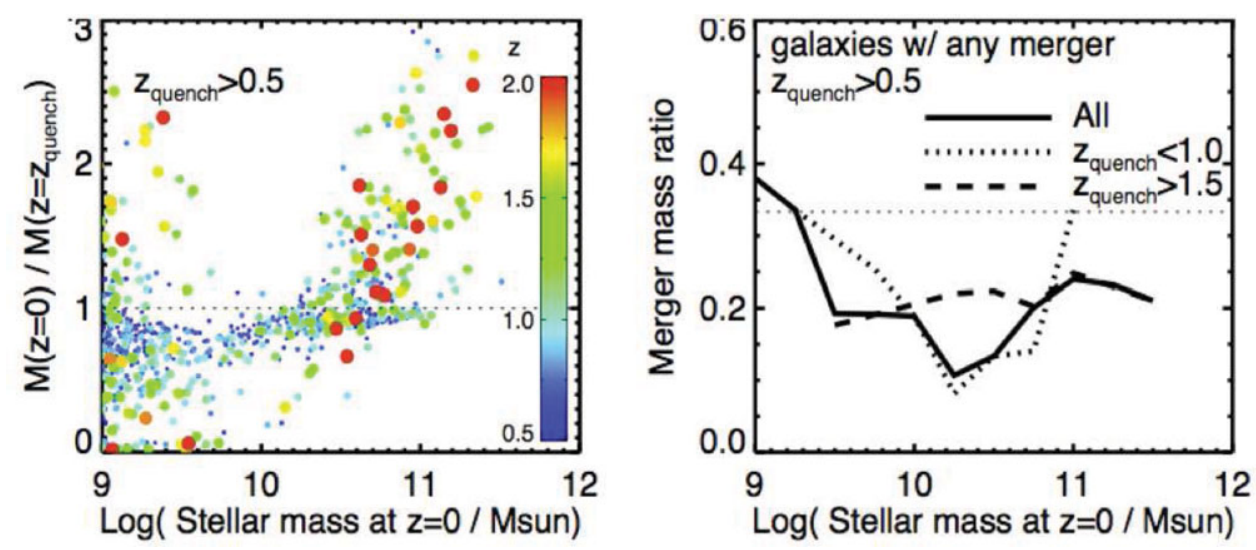

Figure 2. After becoming red, galaxies grow significantly via minor mergers. Left: Mass growth of galaxies quenched at $z>0.5$, expressed as the $z=0$ stellar mass divided by the mass at the time of quenching. Galaxies are color-coded by the redshift at which they were quenched. Massive galaxies have typically grown by factors $\sim 2$. Right: Mass-weighted mean merger mass-ratio for galaxies quenched at $z>0.5$. Dashed line at 0.33 shows the typical division between major and minor mergers $(1: 3)$. Quenched galaxies grow mostly via minor $(\sim 1: 5)$ mergers. Taken from Gabor \& Davé (2012).

\section{Hot gas quenching explains both "mass quenching" and "environment quenching"}

In the hot halo quenching model, any galaxies that live in a hot corona are starved of incoming fuel for star-formation. A galaxy has two alternative paths to live inside a hot halo: a) it is the central galaxy in a halo of $>10^{12} M_{\odot}$, where a hot coronae is likely to form; or b) it is a satellite galaxy in such a halo. Case (a) can be thought of as "mass quenching" or "central quenching", and case (b) can be thought of as "environment quenching" or "satellite quenching."

These two modes of quenching are apparent in Figure 3, inspired by Peng et al. (2010) and taken from Gabor \& Davé (2012). The fraction of red galaxies increases with overdensity (i.e. in denser environments) and with stellar mass. Moreover, the "boxy" shape of the contours suggests that these modes are independent. In our simulation, they both result from hot gas cutting off the fuel supply for star-formation.

"Central quenching" and "satellite quenching" can be explained naturally in this model. In hydrodynamic simulations, halos above $\sim 10^{12} M_{\odot}$ are all dominated by hot gas (Birnboim \& Dekel 2003, Kereš et al. 2005, Gabor et al. 2010). Furthermore, in the absence of quenching, stellar mass closely tracks halo mass. So a star-forming galaxy will increase its stellar mass as its halo mass increases due to accretion. Then, when the halo reaches $\sim 10^{12} M_{\odot}$ (i.e. stellar mass reaches $\sim 10^{10.5} M_{\odot}$ ), a hot corona will form which quenches star-formation. This manifests as a strong increase in red galaxy fraction at stellar masses $\gtrsim 10^{10.5} M_{\odot}-$ mass quenching.

Once a massive galaxy is quenched in this way, its satellite galaxies will also be quenched since they live in the same hot halo. The dark matter halo will continue to grow as additional galaxies fall in. Such infalling galaxies typically start out as starforming centrals, but after becoming satellites they will be quenched by the hot gas halo. This is satellite quenching. Satellites become quenched regardless of their masses, as long as they live in sufficiently dense environments where hot gas dominates. 


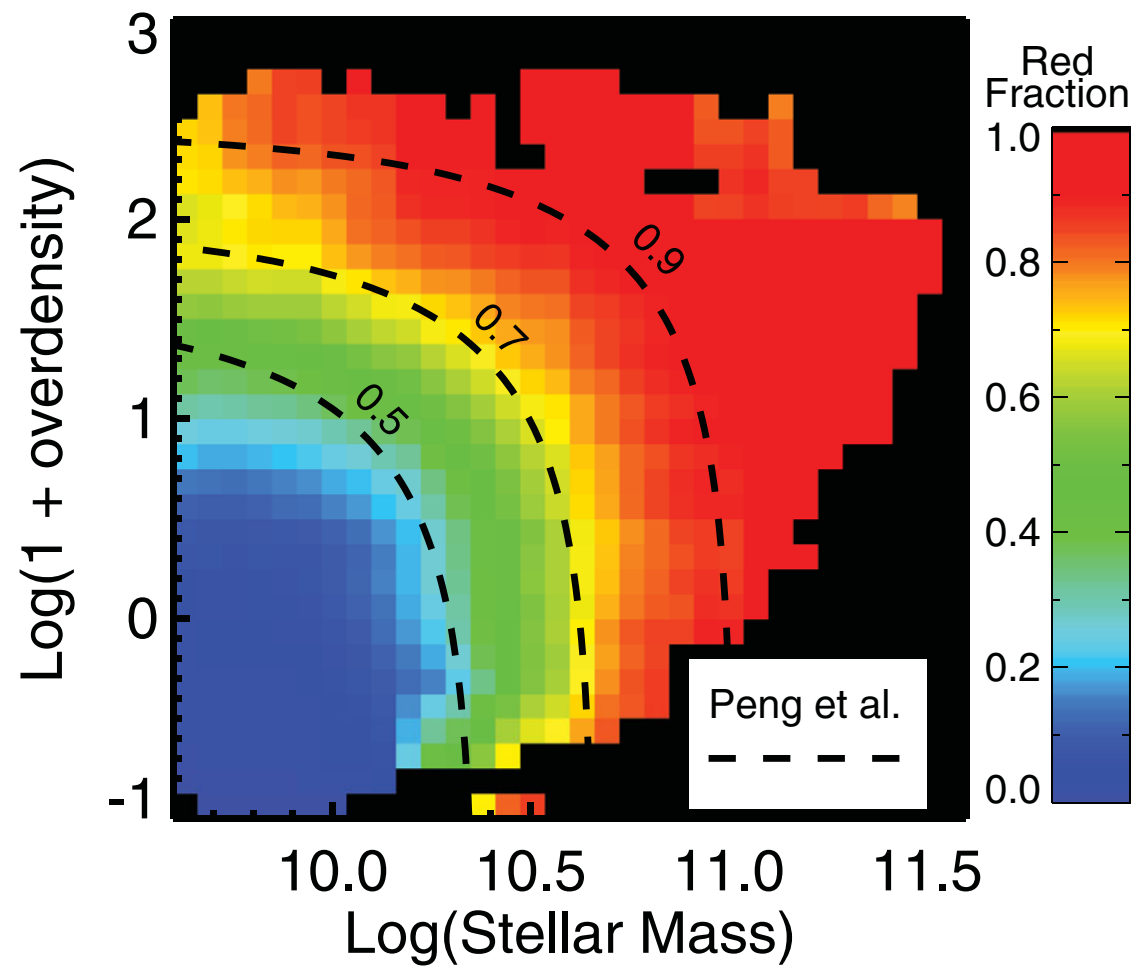

Figure 3. Galaxies are quenched at high stellar masses and high overdensities. I show the red fraction (color-coding) of galaxies as a function of stellar mass and local overdensity, a measure of environment. Dashed lines are from the model of Peng et al. 2010. The boxy contour shape suggests that "environment quenching" and "mass quenching" are independent, when in fact they both result from the presence of hot gas. Taken from Gabor \& Davé (2012).

\section{Summary}

Despite its simplicity, the hot halo quenching model does a remarkable job of matching basic observables. Although hot halos do not tell the whole story, they appear to be the dominant factor in forming the red sequence. Mergers must play some role in the formation of today's red ellipticals, but halting the inflow of new gas is crucial.

\section{References}

Birnboim, Y. \& Dekel, A. 2003, MNRAS, 345, 349

Bower, R. G. et al. 2006, MNRAS, 370, 645

Croton, D. et al. 2006, MNRAS, 365, 11

Gabor, J. M., Davé, R., Finlator, K., \& Oppenheimer, B. D. 2010, MNRAS, 407, 749

Gabor, J. M., Davé, R., Oppenheimer, B. D., \& Finlator, K. 2011, MNRAS, 417, 2676

Gabor, J. M. \& Davé, R. 2012, MNRAS, 427, 1816

Hopkins, P. F., Cox, T. J., Kereš, D., \& Hernquist, L. 2008, ApJS, 175, 390

Kereš, D., Katz, N., Weinberg, D. H., \& Davé, R. 2005, MNRAS, 363, 2

Oser, L., Naab, T., Ostriker, J. P., \& Johansson, P. H. 2012, ApJ, 744, 63

Peng, Y.-j. et al. 2010 ApJ, 721, 193

Somerville, R. S., Hopkins, P. F., Cox, T. J., Robertson, B. E., \& Hernquist, L. 2008, MNRAS, 391,481 